\title{
New all-optical RIN suppressing, image rejection receiver with efficient use of LO- and
} signal-power

Pedersen, Rune Johan Skullerud; Ebskamp, F.

Published in:

I E E E Photonics Technology Letters

Link to article, DOI:

$10.1109 / 68.262574$

Publication date:

1993

Document Version

Publisher's PDF, also known as Version of record

Link back to DTU Orbit

Citation (APA):

Pedersen, R. J. S., \& Ebskamp, F. (1993). New all-optical RIN suppressing, image rejection receiver with efficient use of LO- and signal-power. I E E E Photonics Technology Letters, 5(12), 1462-1464.

https://doi.org/10.1109/68.262574

\section{General rights}

Copyright and moral rights for the publications made accessible in the public portal are retained by the authors and/or other copyright owners and it is a condition of accessing publications that users recognise and abide by the legal requirements associated with these rights.

- Users may download and print one copy of any publication from the public portal for the purpose of private study or research.

- You may not further distribute the material or use it for any profit-making activity or commercial gain

- You may freely distribute the URL identifying the publication in the public portal 


\title{
New All-Optical RIN Suppressing, Image Rejection Receiver with Efficient Use of LO- and Signal-Power
}

\author{
R. J. S. Pedersen and F. Ebskamp
}

\begin{abstract}
We propose an all-optical method of achieving suppression of local-oscillator (LO) RIN, image rejection, and efficient use of signal and $L O$ power for a heterodyne receiver. Hence, only one photodetector is needed compared to four in the conventional optical-electrical image rejection receiver.
\end{abstract}

\section{INTRODUCTION}

$\mathbf{M}$ ULTI-CHANNEL coherent optical systems are expected to play an important role in future highcapacity optical networks where efficient use of the optical bandwidth is sought. The task will then be to minimize the interchannel spacing, and this leads to the use of image rejection techniques [1].

In a coherent optical receiver, the intensity fluctuations of the local oscillator (LO) light (relative intensity noise (RIN)) can degrade the system performance significantly, since it prevents the receiver from operating in the shot noise limit regime [2]. The conventional way to reduce LO-RIN is to use a balanced receiver configuration. Schematically, this is shown in Fig. 1 in the form of a conventional optical-electrical balanced image rejection receiver.

This configuration has the advantage of efficiently using all LO and signal power, suppressing LO-RIN and allowing a narrow interchannel spacing due to rejection of the image channel. The cost of these advantages is the need for four photodetectors that have to be matched. Furthermore, optical and electrical path lengths have to be matched, which is difficult at high frequencies.

\section{All-Optical Setup}

In this paper, an all-optical method of achieving efficient use of LO and signal power, suppression of LO-RIN, and rejection of the image channel is presented. The setup is quite simple as shown in Fig. 2. Note that only one photodetector is necessary. The function of the Mach-Zehnder interferometer (MZI) is twofold: First, it

Manuscript received July 13, 1993; revised August 27, 1993. This work was supported by the Danish Technical Research Council.

The authors are with the Center for Broadband Telecommunications, Electromagnetics Institute, Technical University of Denmark, DK-2800 Lyngby, Denmark.

IEEE Log Number 9214118.

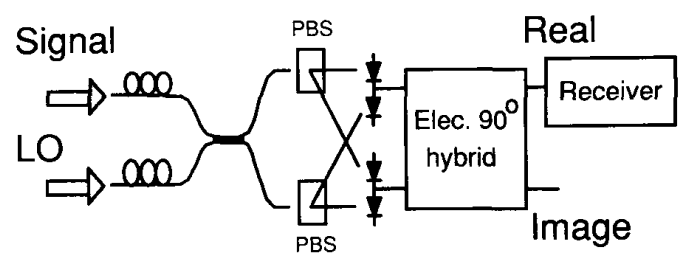

Fig. 1. Conventional optical-electrical balanced image rejection receiver (PBS: polarization beam splitter).

is used as a multiplexer and by selection of a free spectral range (FSR), which equals two times the IF-frequency of the receiver, it is ensured that all power from both the signal branch and the LO branch will exit the same output port of the MZI. Second, when the first criterion is met, it is inherent in the setup that the LO-RIN is suppressed in the IF-band, since the amplitude noise of the LO in the IF-band will exit the other output port due to the frequency difference between the LO carrier and the noise. The coherence time $t_{c}=1 / \pi \Delta \nu$ of the LO, where $\Delta \nu$ is the linewidth, should be kept much longer than the delay in the interferometer to minimize the noise generated by phase noise to amplitude noise conversion in the interferometer [3]. In most cases, this is fulfilled by the linewidth requirements for proper operation of the heterodyne system itself.

A fiber MZI (two 3-dB couplers in cascade) with an FSR of approximately $15 \mathrm{GHz}$ is used to experimentally verify the transfer function and RIN suppression. The light from one laser is coupled into the signal arm of the interferometer, and the frequency is adjusted to give maximum output of one output arm. The output from this arm is then coupled to an HP71400 lightwave analyzer. Light from another laser is coupled into the LO arm, and the frequency of this second laser is swept over a range corresponding to an IF-frequency of $0-22 \mathrm{GHz}$. The recorded beat-signal is shown in Fig. 3 (thin line). The theoretical transfer function for the interferometer is shown as the thick line.

Fig. 3 shows that a maximum occurs when the frequency difference between signal and LO equals FSR/2, which is then the ideal IF-frequency for this particular MZI. 


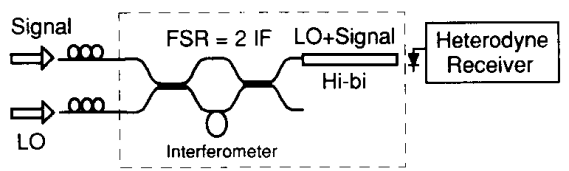

Fig. 2. All-optical, RIN suppressing and image rejecting receiver.

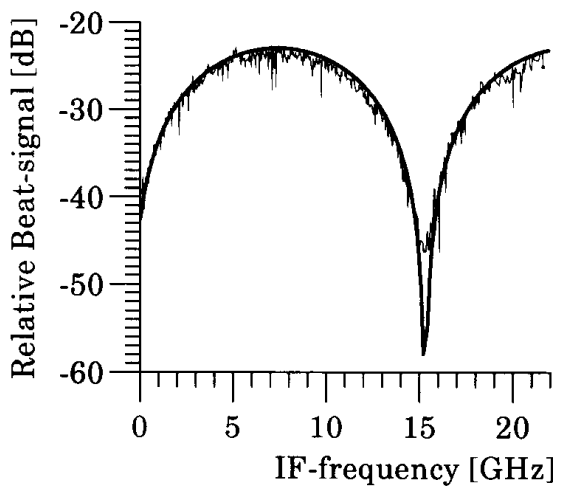

Fig. 3. Max hold trace of beat-signal (thin line) and theoretical curve (thick line).

To verify the LO-RIN suppression, the RIN is measured for the light directly from the LO (Fig. 4(a), solid line) and after passing the MZI (Fig. 4(a), dashed line). To highlight the action of the interferometer, the difference between the curves is shown in Fig. 4(b) (thin line), where $1 \mathrm{~dB}$ of insertion loss has been assumed. The theoretical LO-RIN suppression is shown as the solid line.

The curve shown in Fig. 4(b)represents the filtering of the interferometer, and it is seen that the maximum suppression is at FSR/2, which again matches the ideal IF-frequency. Hence, by the application of the interferometric coupling, both efficient use of signal and LO power as well as suppression of LO-RIN has been achieved.

It is important to note that no requirements have been put onto the polarization of either the signal or the LO so far. Hence, the polarizations of signal and LO can be adjusted freely, and this allows the use of the previously reported [4] all-optical image-rejection mixer. By applying a piece of $\mathrm{Hi}-\mathrm{Bi}$ fiber with a length corresponding to a transit time-delay difference between the two principal axes that equals 0.5 times the MZI delay and adjusting the polarization of the signal and LO such that they are equal at the output of the $\mathrm{Hi}-\mathrm{Bi}$ fiber, it is at the same time ensured that the image channel will suffer from low heterodyning efficiency [4]. The LO-RIN will actually also experience a further reduction of about $3 \mathrm{~dB}$ due to the filtering of the optical image-rejection mixer. The imagerejection part does not need any stabilization in itself, since both signal and LO will experience the same change, if any, as described in [4], but the input polarizations should be maintained as always in a heterodyne receiver. The MZI should be stabilized with respect to the LO by regulating the interferometer delay. The heterodyne re-

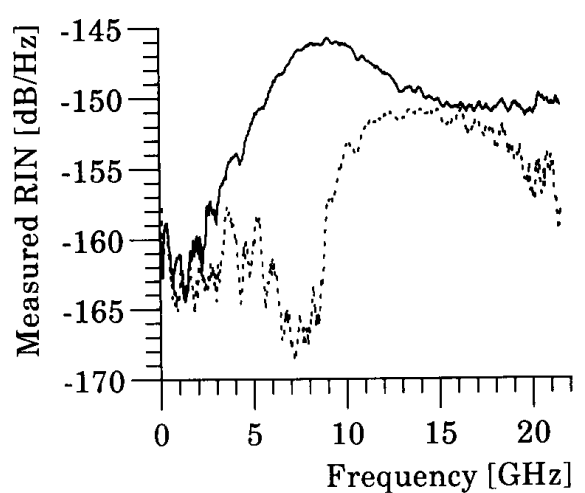

(a)

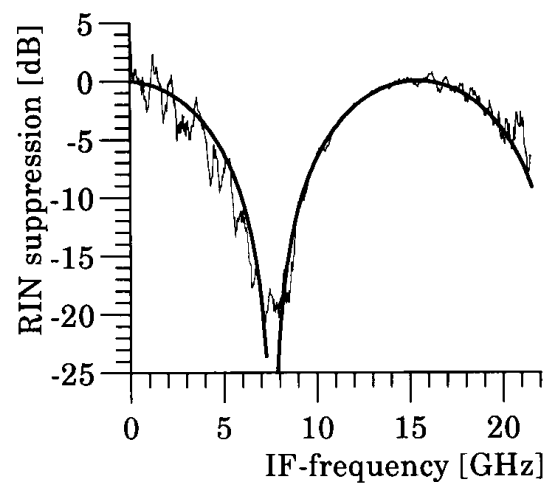

(b)

Fig. 4. (a) Measured RIN spectra for no interferometer (solid line) and through interferometer (dashed line). (b) Difference between curves of Fig. 4(a) (thin line) and theoretical RIN suppression (thick line).

ceiver will lock the LO to the signal, which will stabilize the whole scheme. This also ensures that the LO can be tuned to a new channel while the MZI remains locked to the LO. Furthermore, locking the MZI to the LO also guarantees that a lock signal is always present, and this is important when the proposed receiver is used in a polarization diversity configuration.

The LO-RIN suppression of the all-optical part is not decreased significantly even if the FSR $/ 2$ of the MZI is chosen to be $10 \%$ off the ideal value. This is shown in Fig. 5 , where the theoretical LO-RIN suppression is calculated as a function of the channel bandwidth relative to the IF-frequency. In this calculation, a uniform LO-RIN spectrum is assumed. When FSR $/ 2$ does not match the IF-frequency, a transmission loss of the LO results; but for a deviation of $10 \%$, it amounts to less than $0.2 \mathrm{~dB}$. For an LO position different from maximum transmission, however, the requirements to the LO linewidth increase [3], since the phase noise to amplitude noise is no longer at its minimum point. For a relative bandwidth of $50 \%$ and ideal FSR/2, a LO-RIN suppression of more than 15 $\mathrm{dB}$ is found. 


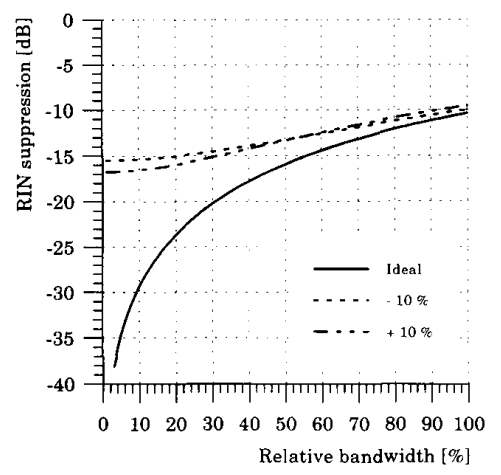

Fig. 5. Calculated RIN suppression as a function of the signal bandwidth relative to the IF-frequency.

The suppression of the image channel is also calculated and shown in Fig. 6 as a function of the channel bandwidth relative to the IF-frequency. Also in this case, the influence of a $10 \%$ deviation of the MZI FSR is shown, and a uniform image channel spectral density has been assumed.

Only a small influence of the MZI FSR can be seen. For a relative bandwidth of $50 \%$, an image channel suppression of almost $20 \mathrm{~dB}$ is found.

\section{CONCLUSION}

We have presented a new all-optical method of achieving a heterodyne signal where the LO-RIN and the image channel have been suppressed while efficient use of both LO and signal power is made. This is achieved with only one photodetector, compared to four for the conventional optical-electrical configuration. A $15-\mathrm{dB}$ reduction of

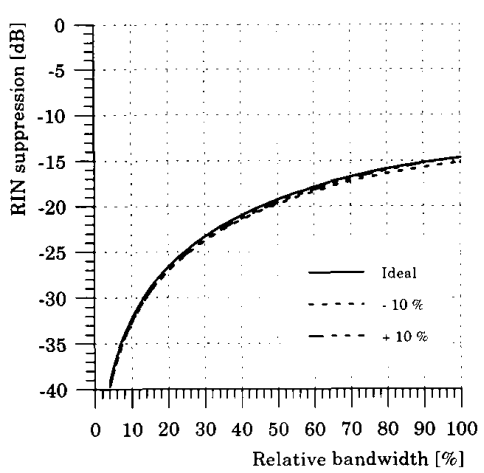

Fig. 6. Calculated image rejection as a function of the signal bandwidth relative to the IF-frequency.

LO-RIN can be obtained for a relative channel bandwidth of $50 \%$. The image channel of the same bandwidth is suppressed by almost $20 \mathrm{~dB}$.

\section{ACKNOWLEDGMENT}

The authors are grateful for fruitful discussions with C. Mahon and P. Jeppesen.

\section{REFERENCES}

[1] B. S. Glance "An optical heterodyne mixer providing image frequency rejection," J. Lightwave Technol., vol. LT-4, no. 11, pp. $1722-1725,1986$.

[2] G. L. Abbas, V. W. S. Chan, and T. K. Yee, "A dual-detector optical heterodyne receiver for local oscillator noise suppression," J. Lightwave Technol., vol. LT-3, no. 5, pp. 1110-1122, 1985.

[3] K. Petermann, Laser Diode Modulation and Noise. New York: Kluwer, 1988, ch. 8.

[4] C. Mahon, G. Schiellerup, and R. J. S. Pedersen, "Simple, all-fibre optical image-rejection mixer for optical heterodyne receivers," in Proc. 16th European Conf. Opt. Commun., 1990, vol. 3, ThG2.2, pp. 1029-1032. 